\title{
Double Colorectal Cancer Only Diagnosed by Computed Tomographic Colonography
}

\author{
Koichi Nagata Shungo Endo Kishiko Tatsukawa \\ Shin-ei Kudo
}

Digestive Disease Center, Showa University Northern Yokohama Hospital, Yokohama, Japan

\section{Key Words}

Colorectal cancer · Obstruction · Computed tomography · Colonography · Virtual colonoscopy

\begin{abstract}
A 58-year-old woman presented to her physician with rectal bleeding and intermittent diarrhea. Optical colonoscopy revealed a bulky tumor which was diagnosed as rectal cancer. She was referred to our institution for further evaluation and treatment. Slim optical colonoscopy showed an obstructive cancer in the rectosigmoid junction and no information of the proximal side of the obstruction. The patient then underwent computed tomographic (CT) colonography for further evaluation of the proximal side. Three-dimensional endoluminal 'fly-through' images revealed another protruded lesion in the proximal side of the obstruction. Diagnosis of synchronous double cancer was made by CT colonography. This CT data was not only used to create three-dimensional images but also to decide on a preoperative clinical staging. Laparoscopy-assisted high anterior resection was performed and $\mathrm{T} 3$ rectal cancer and $\mathrm{T} 1$ sigmoid colon cancer were confirmed in the resected specimen. Follow-up optical colonoscopy revealed no other tumors. CT colonography has recently become a popular preoperative examination tool with significant improvement in quality of image due to a rapid progress in computer technology. CT colonography correctly showed synchronous double cancer in our case and provided crucial information for planning surgery. We recommend that CT colonography should be used for evaluating the proximal side of obstructive colorectal cancer.
\end{abstract}

\section{Introduction}

In patients with obstructive colorectal cancer, where conventional colonoscopy cannot traverse obstruction, clearance of the proximal colon remains a problem. 


\section{Case Report}

A 58-year-old woman presented to her physician with rectal bleeding and intermittent diarrhea. Conventional colonoscopy revealed a bulky tumor which was diagnosed as rectal cancer. She was referred to our institution for further evaluation and treatment. Slim optical colonoscopy (PCFP240AL/I, Olympus, Tokyo, Japan) showed obstructive cancer in the rectosigmoid junction (fig. 1 , arrow A) and no information of the proximal side of the obstruction. The patient then underwent multidetector-row computed tomographic (CT) colonography (virtual colonoscopy) for further evaluation of the proximal colon. Three-dimensional endoluminal 'fly-through' images revealed another protruded lesion (fig. 2a, arrow B) in the proximal side of the obstruction. Diagnosis of synchronous double cancer was made by CT air-contrast enema image (fig. 2b, arrows A and B). CT colonography showed no other tumors proximal to the obstruction. This CT data was not only used to create threedimensional images but also to decide on a preoperative clinical staging. Laparoscopy-assisted high anterior resection was performed and $\mathrm{T} 3 \mathrm{rectal}$ cancer and $\mathrm{T} 1$ sigmoid colon cancer were confirmed in the resected specimen (fig. 3 , arrows A and B). The postoperative course was uneventful. Follow-up conventional colonoscopy revealed no other tumors.

\section{Discussion}

Between 4.6 and $11 \%$ of patients with colorectal cancer have a second synchronous cancer [1-3]. Preoperative evaluation of the whole colon and rectum in patients with colorectal cancer is widely recommended [4]. Optical colonoscopy is the current gold standard method as it examines the whole colon and rectum. However, preoperative examination by optical colonoscopy may be hampered by occlusive colorectal cancer, and failure to find the presence of a synchronous cancer will change the surgical approach from circumscribed resection to resection of the involved segments [3]. CT colonography might be offered in preference to barium enema for incomplete colonoscopy $[5,6]$. CT colonography has recently become a popular preoperative examination tool with significant improvement in quality of image due to a rapid progress in computer technology $[7,8]$. CT colonography correctly showed synchronous double cancer in our case and provided crucial information for planning surgery. We recommend that CT colonography should be used for evaluating the proximal side of obstructive colorectal cancer, as it is well tolerated and less invasive $[6,9]$. 
Fig. 1. Optical colonoscopy showing an obstructive cancer in the rectosigmoid junction (arrow A).

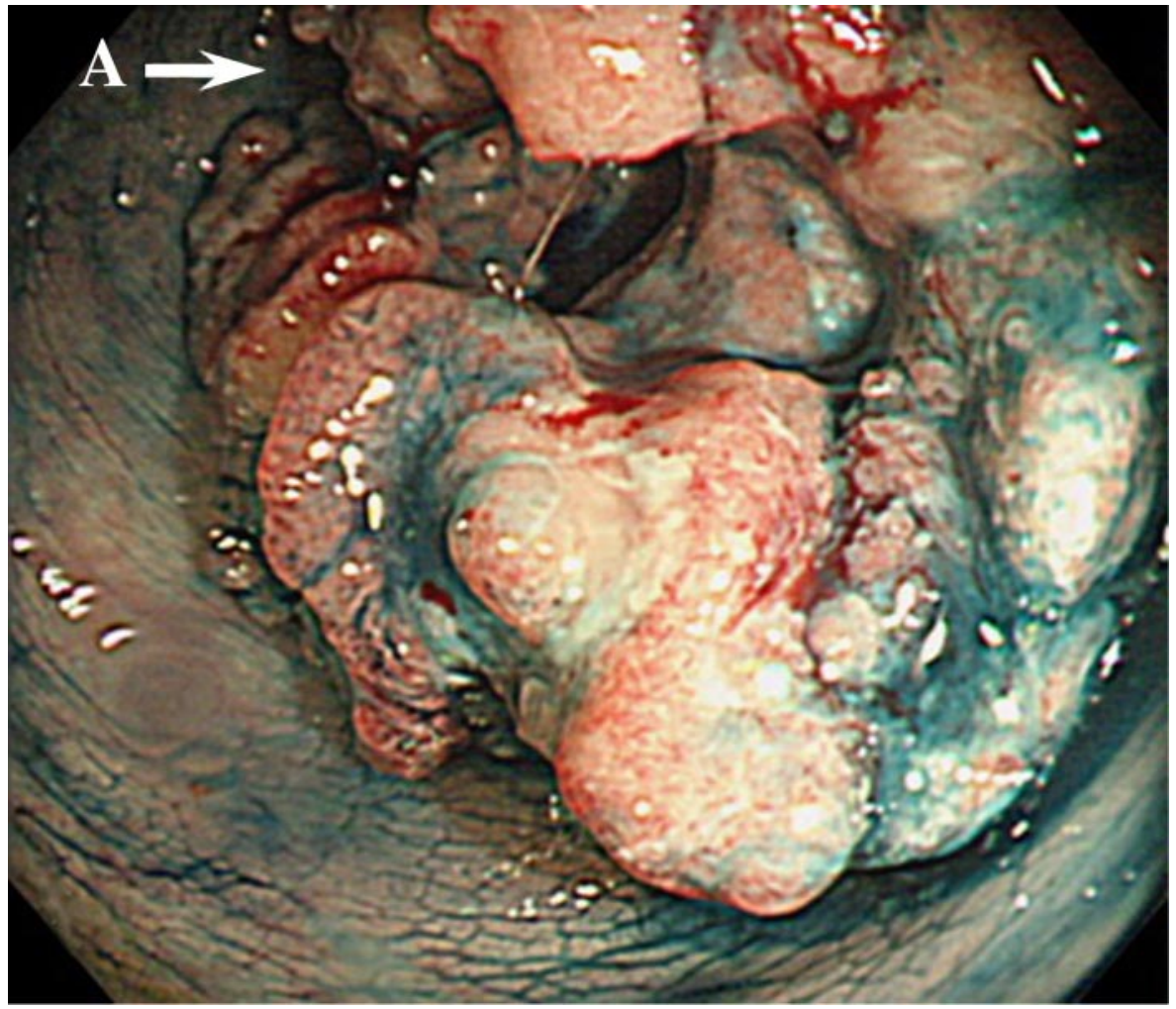

Fig. 2. CT colonography images. a Fly-through image revealing another protruded lesion (arrow B) proximal to the obstruction. $\mathbf{b}$ CT air-contrast enema image showing synchronous double colon cancer (arrows A and B).
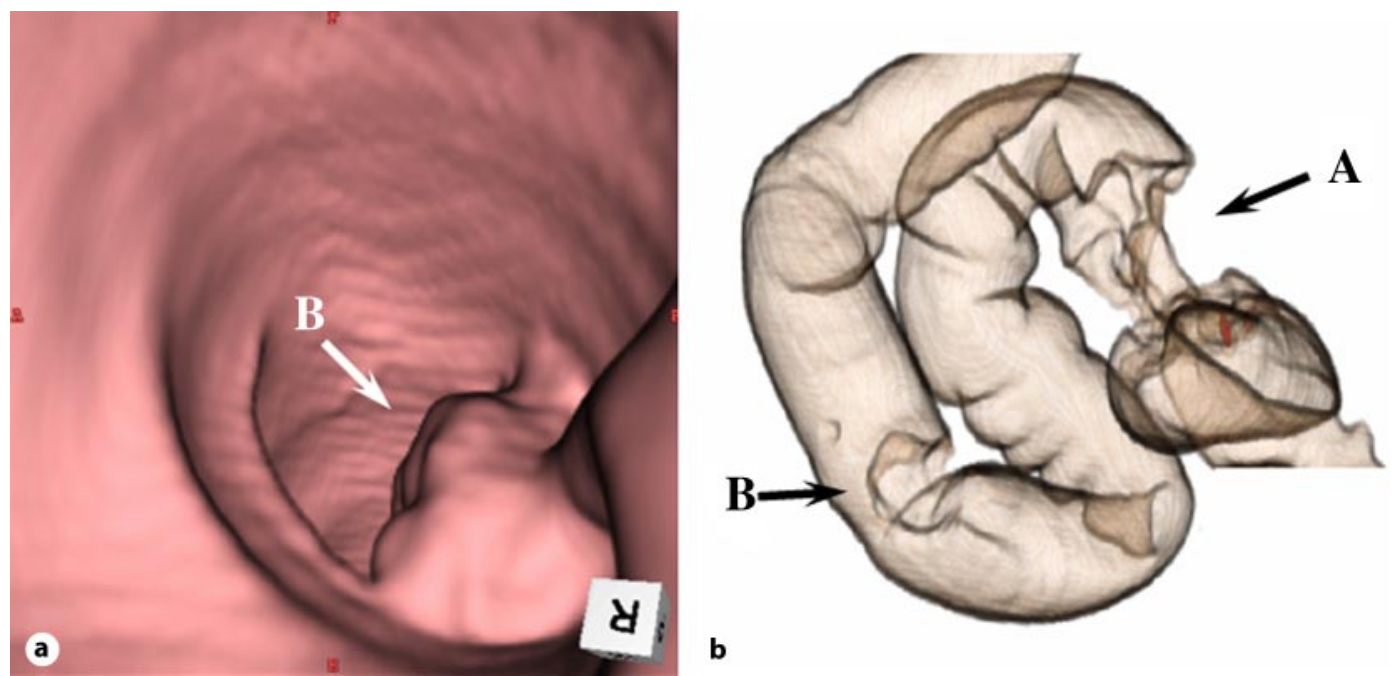
Fig. 3. Resected specimen showing T3 rectal cancer (arrow A) and T1 sigmoid colon cancer (arrow B).

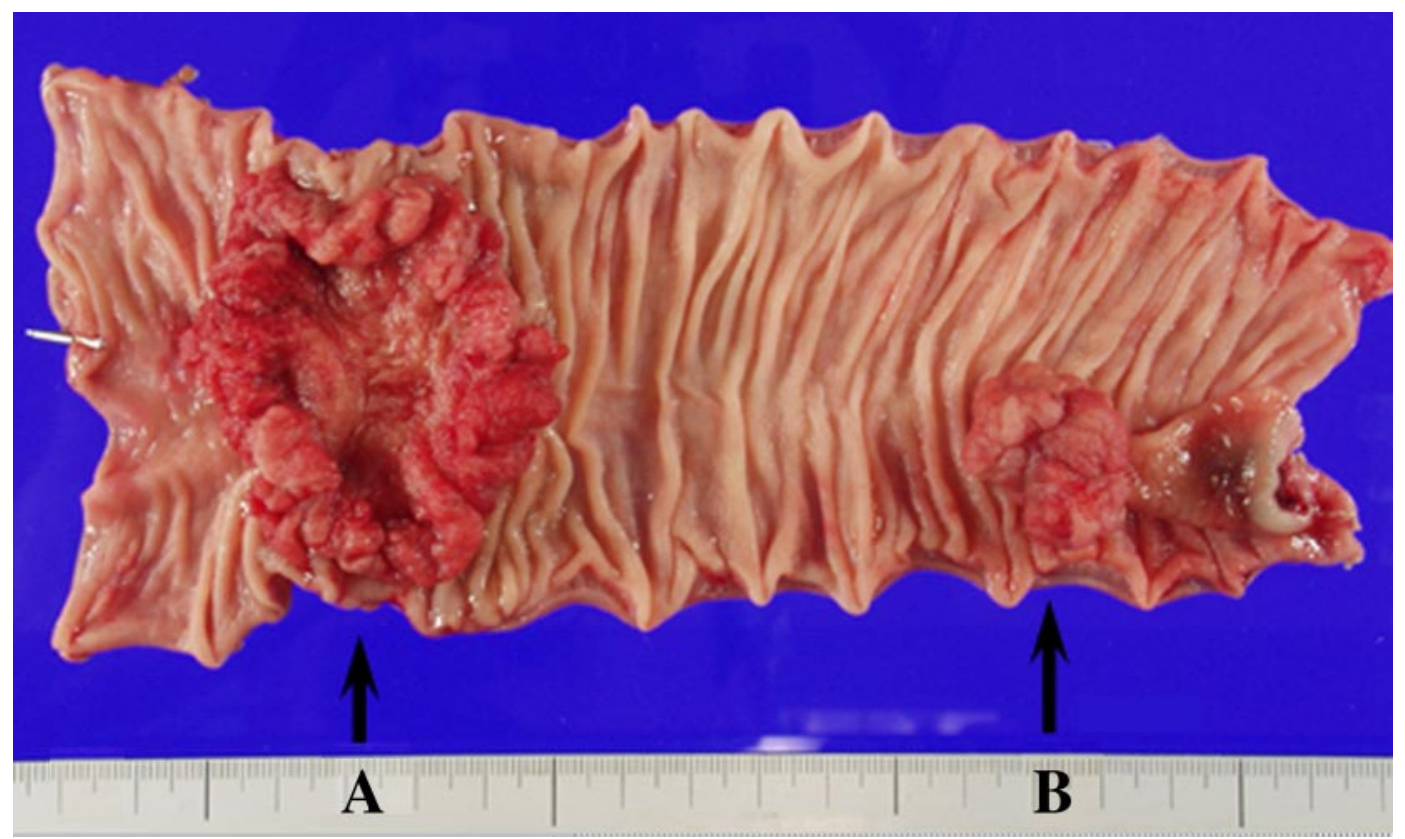




\section{References}

1 Ekelund GR, Pihl B: Multiple carcinomas of the colon and rectum. Cancer 1974;33:1630-1634.

-2 Cunliffe WJ, Hasleton PS, Tweedle DE, Schofield PF: Incidence of synchronous and metachronous colorectal carcinoma. Br J Surg 1984;71:941-943.

-3 Hennekinne-Mucci S, Tuech JJ, Brehant O, Lermite E, Bergamaschi R, Pessaux P, Arnaud JP: Emergency subtotal/total colectomy in the management of obstructed left colon carcinoma. Int J Colorectal Dis 2006;21:538-541.

-4 Dasmahapatra KS, Lopyan K: Rationale for aggressive colonoscopy in patients with colorectal neoplasia. Arch Surg 1989;124:63-66.

5 Fletcher RH: The end of barium enemas? N Engl J Med 2000;342:1823-1824.

6 Neri E, Giusti P, Battolla L, Vagli P, Boraschi P, Lencioni R, Caramella D, Bartolozzi C: Colorectal cancer: role of CT colonography in preoperative evaluation after incomplete colonoscopy. Radiology 2002;223:615-619.

7 Nagata K, Endo S, Kudo S, Kitanosono T, Kushihashi T: CT air-contrast enema as a preoperative examination for colorectal cancer. Dig Surg 2004;21:352-358.

8 Nagata K, Endo S, Ichikawa T, Dasai K, Moriya K, Kushihashi T, Kudo SE: Polyethylene glycol solution (PEG) plus contrast medium vs PEG alone preparation for CT colonography and conventional colonoscopy in preoperative colorectal cancer staging. Int J Colorectal Dis 2007;22:69-76.

-9 Copel L, Sosna J, Kruskal JB, Raptopoulos V, Farrell RJ, Morrin MM: CT colonography in 546 patients with incomplete colonoscopy. Radiology 2007;244:471-478. 